\section{Solid-acid Catalyzed Biodiesel Production, Part I: Biodiesel Synthesis from Low Quality Feedstock}

\author{
Dharshini D. Bala ${ }^{\mathrm{a}}$, Mano Misra ${ }^{\mathrm{b}}$, and Dev Chidambaram ${ }^{\mathrm{a}}$ \\ ${ }^{a}$ Department of Chemical and Materials Engineering, University of Nevada Reno, \\ 1664 N. Virginia Street, MS 0388, Reno, NV 89557-0388 \\ ${ }^{b}$ Department of Chemical Engineering, University of Utah, Salt lake City, UT 84112-9203 \\ *dcc@unr.edu
}

Abstract

A series of catalysts containing phosphotungstic acid (PTA) supported on periodic mesoporous silica were prepared and evaluated for the production of biodiesel from used cooking oil. The catalytic and physical properties of the prepared catalysts were investigated. Mesoporous catalyst, KIT-5 (Korea Institute of Technology 5) loaded with 26\% PTA showed high activity for single step esterification, resulting in the successful elimination of a second transesterification step. The yield of fatty acid methyl esters was 93\% from used (waste) cooking oil and 100\% from pure palmitic acid. In addition, the solid acid catalyst retained a proportion of its original catalytic activity after repeated recycling steps. Thus, the prepared solid acid catalyst has the potential to be used for the production of biodiesel from feedstock that contain high amounts of free fatty acids.

Keywords: mesoporous; KIT-5; cooking oil; palmitic acid; 


\section{Introduction}

2 Biodiesel forms one of the components of a comprehensive solution to the world's dependency

3 on fossil fuels for transportation (Dovì et al., 2009; Sharma and Singh, 2009). Biodiesel

4 functions in a fashion similar to petroleum diesel (petro-diesel) but results in lower emissions

5 (Wan Ghazali et al., 2015). Just as energy will have to come from various sources in the near

6 future, so should biodiesel feedstock, and hence, the use of a diverse range of agricultural

7 products for production of both fuel and other resources is a welcome approach. Currently,

8 industrial production of biodiesel predominantly utilizes virgin vegetable oil or animal fat

9 feedstock (Kondamudi et al., 2011; Kumar et al., 2011; Leung et al., 2010).

Traditional processes for biodiesel production from oils were typically developed in the early 1940s-50s (Ma and Hanna, 1999). Observations that have been made over the years about the transesterification process include: (i) excess alcohol (greater than 1.6 times the stoichiometric amount) is necessary for the reaction to proceed towards completion (Gerpen, 2005). To make it cost effective, when high FFA feedstocks are used, the reaction could be conducted in two or more steps, where only a part of the alcohol and catalyst are added initially at each step, and the glycerol is separated at the end of every step (Nie et al., 2006), (ii) short or long chained alcohols could be used; examples include methanol, ethanol, propanol, isopropanol, butanol and pentanol (Leung et al., 2010), of which isopropanol is the only branched chain alcohol (Marchetti et al., 2007). (iii) when high amounts of free fatty acids (FFA) are present in the oils or fats, they should be converted to alkyl esters with an acid catalyst. This could be followed by a standard alkali-catalyzed (like $\mathrm{KOH}$ or $\mathrm{NaOH}$ ) transesterification (Meng et al., 2008). Generally, single step transesterification of oils to alkyl esters is kinetically slower than alkali catalyzed reactions (Cho et al.; Sakai et al., 2009).

Used cooking oils (UCO) typically contain 2-7\% FFA, depending upon their usage and animal fats contain 5\% to 30\% FFA (Knothe and Steidley, 2009; Zhang et al., 2003). In comparison, non-generic feedstocks have different properties (Ahmed et al., 2014) and low quality feedstocks like trap grease could approach 100\% FFA content (Ramos et al., 2009). When the FFA level is greater than 5\%, acid catalysts (such as sulfuric acid) could be used to esterify the free fatty acids to esters as shown in the following reaction (Equation 1):

$$
\mathrm{R}_{1}-\mathrm{COOH}+\mathrm{R}_{2}-\mathrm{OH} \leftarrow \rightarrow \mathrm{R}_{1}-\mathrm{COO}-\mathrm{R}_{2}+\mathrm{H}_{2} \mathrm{O}
$$

\begin{tabular}{ll}
\hline CDAB & Cetyldimethyl(ethyl)ammonium bromide \\
KIT-5 & Korea Institute of Technology 5 \\
MCM & Mobil Composition of Matter No. \\
PTA & Phosphotungstic acid \\
SBA-15 & Santa Barbara Amorphous 15 \\
UCO & Used Cooking Oil
\end{tabular}


Palm oil, containing 50.8\% free fatty acids, esterified to methyl esters using this technique resulted in a yield of $97 \%$ but had a residual acid value of about $5 \%$ palmitic acid. The incomplete reaction was probably due to formation of water during the reaction or presence of water in the reactant mixture (Zhang and Jiang, 2008).

The most common catalysts used for biodiesel production are homogeneous catalysts such as $\mathrm{KOH}, \mathrm{NaOH}, \mathrm{H}_{2} \mathrm{SO}_{4}, \mathrm{HCl}$ and $\mathrm{HNO}_{3}$ (Leung and Guo, 2006). On the other hand, the approach based on the use of heterogeneous catalysts provides a promising alternate route for low-cost biodiesel production (Di Serio et al., 2007; Nair et al., 2012; Tang et al., 2013). Ali Alsalme et al. studied the use of heteropoly acids as catalysts for liquid-phase esterification of hexanoic acid (Alsalme et al., 2008). Their catalytic activity which was measured by the turn over frequency both in homogeneous and heterogeneous catalyst systems was found to be higher than that of the conventional acid catalysts such as sulfuric acid (Alsalme et al., 2008). When solid-acid catalysts replace liquid acids, corrosion and environmental problems linked with the latter can also be avoided and the product purification protocols can be significantly reduced, thereby simplifying biodiesel production and reducing cost.

Properties of solid acid catalysts strongly depend upon the nature and concentration of the acids loaded (Singh and Patel, 2014). Catalyst loading could be evaluated by measuring acidity or by determining the concentrations of the elements present in the solid acid loaded. The determination of elemental concentration in catalysts is usually done by inductively coupled plasma (ICP) spectroscopy since it has very low detection limits (ppm to ppb levels), high sensitivity, and reduced matrix effects due to the high temperature of the argon plasma (Lynch, 2003). Solid samples have to be digested with concentrated acids or bases and the final solution is homogeneous and not affected by initial sample heterogeneities (López-Suárez et al., 2008). Several studies have previously employed ICP for the determination of the per cent loading of the solid acid loaded with the framework (Tan et al., 2012).

26 This two-part study aims to investigate the use of solid-acid loaded mesoporous silica framework as a catalyst for the production of biodiesel from used cooking oil and to understand the kinetics of the methyl esterification to elucidate a reaction mechanism. Phosphotungstic acid has the strongest Brønsted acidity in the HPAs series and has been used in a wide variety of applications such as oxidation, alkylation, esterification and hydrolysis (Kozhevnikov, 1998). Recently, interesting studies focusing on phosphotungstic acid (PTA) functionalized catalysts have been published that demonstrate the vast potential of PTA in catalysis (Jagadeeswaraiah et al., 2010; Singh and Patel, 2014).

This study (Part-I) reports on the use of a mesoporous organosilica, loaded with varying concentrations of phosphotungstic acid (PTA), for the esterification of palmitic acid (model compound) and high FFA containing used cooking oil. The mesoporous material chosen was KIT-5 (Kleitz et al., 2003). KIT-5 possesses highly ordered cage type mesoporous structure with 
specific pore volume. Also, experiments conducted with low FFA containing oils showed that the proposed acid catalyst works effectively with oils containing high FFA.

\section{Experimental}

\subsection{Catalyst preparation}

KIT-5 was prepared in the lab using previously established procedure (Kleitz et al., 2003). PTA was loaded on to this framework at various concentrations ranging between $0 \mathrm{wt} \%$ to $40 \mathrm{wt} \%$. Supported PTA catalysts were prepared by aqueous incipient wetness impregnation onto mesoporous silica i.e. by suspending a known mass of an aqueous solution of PTA (i.e. " $\mathrm{x}$ " $\mathrm{g}$ of PTA in $20 \mathrm{~mL}$ distilled water) per $1 \mathrm{~g}$ of KIT-5 support. This mixture was stirred for $2 \mathrm{~h}$ at room temperature and was followed by evaporation to dryness in a rotary evaporator. The dried samples were then maintained at $100{ }^{\circ} \mathrm{C}$ for $12 \mathrm{~h}$ in an oven, further powdered and calcined at $600{ }^{\circ} \mathrm{C}$ in air for $4 \mathrm{~h}$. All chemicals were purchased from Sigma Aldrich ${ }^{\circledR}$ and were analytical or better grade.

\subsection{Catalyst characterization}

The specific surface area was measured using an Autosorb $2010^{\mathrm{TM}}$ gas sorption analyzer based on the nitrogen adsorption/desorption multipoint Brunauer-Emmett-Teller (BET) method. Morphology was characterized using scanning electron microscopy (SEM). SEM images were obtained at $20 \mathrm{kV}$. Crystalline nature of PTA in the framework was verified using X-ray diffraction (XRD). XRD patterns were obtained using PANalytical X'Pert Pro X-ray diffractometer with $\mathrm{Cu} \mathrm{K} \alpha$ radiation. The mesoporous nature of the organosilica framework was confirmed using BET adsorption isotherms. Catalyst chemistry was confirmed using Fourier transform infrared (FTIR) spectroscopy. A Perkin Elmer inductively coupled plasma optical emission spectrometer (ICP-OES) was employed for the determination of the concentration of the active element, tungsten. The wavelength used was $207.912 \mathrm{~nm}$ and the integration time was $12 \mathrm{~s}$. The catalysts are denoted as PTA-A, PTA-B, PTA-C and PTA-D where A, B, C and D characters represented the various concentrations of PTA loaded on the KIT-5 framework, as explained in Table 1. The $\mathrm{W}$-ion concentration was used to calculate the amount of acid loaded onto the framework. For this purpose, $0.1 \mathrm{~g}$ of the PTA-D catalyst was accurately measured and digested with $15 \mathrm{~mL}$ of $5 \mathrm{M} \mathrm{NaOH}$ solution for $4 \mathrm{~h}$. The solution was then diluted to a known volume and was analyzed for tungsten using ICP-OES. The mass of PTA that leaches from the KIT-5 framework material into the reaction mixture was also evaluated using ICP-OES analyses. For this purpose, $1 \mathrm{~g}$ of the PTA-D catalyst was mixed with $10 \mathrm{~mL}$ of triple distilled water and was refluxed for $3 \mathrm{~h}$. The leachate was then analyzed for tungsten ion concentration.

\subsection{Reaction procedure}

UCO was obtained from a local restaurant. The first step in the processing of UCO was to remove the insoluble materials, such as meat, fries and particles, using a strainer. Prior to 
esterification, $\mathrm{UCO}$ was heated to $100{ }^{\circ} \mathrm{C}$ to lower the water content, if any. Temperature was then maintained at $70{ }^{\circ} \mathrm{C}$. Pure palmitic acid was used as a control. Palmitic acid was allowed to melt and no further processing step was required. Esterification was conducted in a $100 \mathrm{~mL}$ round bottom flask equipped with a magnetic stirrer. $1.5 \mathrm{wt} \%$ of catalyst was added to $10 \mathrm{~g}$ of feedstock. Feedstocks were either UCO or palmitic acid. The oil to alcohol ratio was 1:2 v/v and 1:6 v/v for UCO and palmitic acid respectively. A higher amount of alcohol was used to assist with phase miscibility issues when palmitic acid was employed as a feedstock. It is also assumed to drive the reaction towards completion. However, any excess unused $\mathrm{MeOH}$ at the end of the reaction was recycled and re-used. At the end of $4 \mathrm{~h}$, the biodiesel was obtained after draining off the glycerol and the excess methanol was recovered using a roto-evaporator. The product was then washed gently with warm water as a purification step.

For recycling studies, the reaction was stopped after $4 \mathrm{~h}$ and the resulting products were transferred to a separatory funnel. The catalyst was recovered and washed with hexane. It was further dried at $100{ }^{\circ} \mathrm{C}$ to remove any water molecules that could affect its catalytic activity. The resulting oil-free spent catalyst powder was then reused as catalyst for a new reaction under the same conditions. Methanol was recovered from the biodiesel product using a rotary evaporator and reused for subsequent experiments. The amount of methanol recovered was close to $5 \mathrm{~mL}$ which is a part of the excess $\mathrm{MeOH}$ used for driving the reaction forward.

\subsection{Analytical methods}

The AOCS official method CA 5a-40 (AOCS, 1998) was used to analyze the FFA content of oils. High-performance liquid chromatography (HPLC, Shimadzu LC-10A) equipped with an ultraviolet photometric detector was used for the analysis of the product samples. A C18 Allure column was used for the separation. The mobile phase B was a mixture of isopropanol and hexane in the volumetric ratio of 5:4, and the mobile phase A was 99.9\% HPLC grade methanol. The flow rate of the mobile phases was $0.5 \mathrm{mLmin}^{-1}$, and the column temperature was set at 40 ${ }^{\circ} \mathrm{C}$. The components identified by the HPLC include triglycerides, diglycerides, monoglycerides, methyl esters, and free fatty acids. The identities and relative proportions of fatty acids in the methyl ester product were determined using a Shimadzu gas chromatography-mass spectrometry (GC-MS) QP 2010 using splitless injection. This was equipped with a SHR-5XLB capillary column (25 mm thickness x $30 \mathrm{~m}$ length) and had electron ionization detector $(70 \mathrm{eV})$ using helium as a reagent gas. A temperature gradient was selected so that the temperature rose from $100{ }^{\circ} \mathrm{C}$ to $240{ }^{\circ} \mathrm{C}$ at the rate of $1.5{ }^{\circ} \mathrm{Cmin}^{-1}$.

\section{Results and Discussion}

\subsection{Acid exchange and surface area of catalysts}

All catalysts used in this study were calcined. Table 1 reports the amount of acid in wt $\%$ that was successfully loaded on the framework and the surface area of the PTA-loaded KIT-5 catalyst. Unloaded KIT-5 had the highest surface area at $978 \mathrm{~m}^{2} \mathrm{~g}^{-1}$. Among the PTA loaded KIT-5 
catalysts, the catalyst with the least amount of PTA loading, namely PTA-A (with $~ 4.1 \%$ loading) had the highest surface area due to lesser pore blockage by the loaded phosphotungstic acid. An increase in solid-acid loading is expected to result in lower surface area but also increased yield and conversion.

\subsection{FTIR, SEM and XRD analysis}

FTIR spectra corresponding to KIT-5 framework, PTA loaded on KIT-5, and framework free PTA is shown in Figure 1. The vibrations of asymmetrical/symmetrical stretching at 1633, 1080 and $816 \mathrm{~cm}^{-1}$ corresponds to Si-O-Si bonds (Peña-Alonso et al., 2002). Also the feature at 949 $\mathrm{cm}^{-1}$ relates to $\mathrm{Si}-\mathrm{OH}$ vibrations of surface silanols which is a typical characteristic of mesoporous silica (Wang et al., 2008). Pure PTA shows the typical IR absorption at approximately $1080(\mathrm{P}-\mathrm{O}), 982$ (terminal $\mathrm{W}=\mathrm{O}), \sim 897(\mathrm{~W}-\mathrm{O}-\mathrm{W}) \mathrm{cm}^{-1}$ which are asymmetric vibrations associated with typical Keggin anions (Staiti et al., 1999). A covalent bonding peak was observed at $726 \mathrm{~cm}^{-1}$ and is assumed to be a stretching vibration of W-O bonds which is usually observed at $730 \mathrm{~cm}^{-1}$ (Hough, 2007). There is a slight shift in the peaks due to the loading of PTA on the framework. The higher activity of PTA supported catalysts compared to plain support could be due to the Lewis acidity arising due to the stronger interactions between PTA and -OH groups of silica.

Also, the presence of PTA on loaded catalysts was confirmed using SEM and XRD. The SEM images shown in Figure 2a, 2b, 2c, 2d corresponding to PTA-A, PTA-B, PTA-C, and PTAD respectively confirmed the particle size of KIT-5 framework, which is a few $\mu \mathrm{m}$ (Mushrush $e t$ $a l ., 1991)$. The exact particle diameter of PTA is unknown but is estimated to be in the 10-20 nm range. The investigated KIT-5 contains pores with diameters between $2 \mathrm{~nm}$ and $34 \mathrm{~nm}$. Mesoporous silica materials consisting of interconnected large pore cage type mesoporous system with three-dimensional porous network are advantageous than those consisting of a hexagonal pore structure with a uni-dimensional array of pores. By controlling particle size, very specific pore characteristics can be designed into a product. Pore characteristics often are the key to catalyst performance. In this case, $\mathrm{W}$ ions react with the silica template collapsing/blocking the microporous channels, if any, in the KIT-5 and resulting in the large mesopores and macropores $(17$ and $50 \mathrm{~nm}$ ) in the catalyst obtained. These results indicate that PTA molecules were immobilized in the channels of silica and dispersed in a monolayer on the surface.

The powder XRD patterns of pure PTA, mesoporous calcined KIT-5 and PTA supported on KIT-5 are shown in Figure 3. The peaks are clearly resolved and the incorporation of PTA on KIT-5 is evident. XRD spectra confirm the Keggin structure of PTA. Two small broad diffraction peaks: $2 \Theta=25.3^{\circ}$ and $36.6^{\circ}$ for Keggin structure corresponding to bulk crystal polytungstate (PW) are observed, which indicates that the intact Keggin structure of PW starts to aggregate on the pore walls of KIT-5. Compared with the other porous silica materials, the large pore sizes and high surface area of KIT-5 would accommodate more discrete heteropoly acids. XRD studies on other silica supported phosphotungstic acid materials produced similar 
XRD patterns and confirmed the formation of small crystallites of PTA when higher amounts of acid was loaded and did not show any diffraction peaks with low concentrations of heteropoly acids indicating the well dispersed nature of the heteropoly acid (Bardin and Davis, 2000).

The calcined product was mesoporous and the loaded product retained mesoporosity as indicated by $\mathrm{N}_{2}$ sorption isotherms. According to the classification of the isotherm types by International Union of Pure and Applied Chemistry, the isotherms obtained from KIT-5 and PTA loaded KIT-5 exhibited mesoporous behavior (Type IV/Type V). Also, the data indicates that the mesoporous property of the parent material is not compromised due to addition of PTA (Figure 4). The isotherm displayed no indication of any changes in the mesostructure related to PTA incorporation indicating that the particles were either too small or well dispersed. These results are indicative of a stronger interaction between PTA and silica.

\subsection{Catalyst stability studies}

Leaching of active material from a catalyst is undesirable and thus leaching studies were performed to confirm the stability of the catalysts. Analysis results revealed that $0.0045 \mathrm{~g}$ of PTA had leached from the catalyst framework; this corresponds to $1.5 \%$ of the total mass of PTA $(0.3 \mathrm{~g})$ added to the framework. Although PTA is highly soluble in water, results indicate that a large amount of PTA is still retained on the catalyst following leaching studies. This could be due to the W-O covalent bonding observed in the PTA-D catalyst, as discussed in section 3.2. The results obtained from the leaching experiments confirm the stability of the catalysts used in this study.

\subsection{Yield and conversion}

UCO, canola, and corn oil had a FFA content of $5.94 \%, 0.15 \%$, and $0.17 \%$ as oleic acid, respectively. The corresponding acid numbers were $11.88,0.30$, and 0.34 for UCO, canola, and corn oil, respectively. Palmitic acid was a pure compound, and had a FFA content of $99 \%$ and acid number of 197.0. Reactions were conducted under the same operating conditions in order to evaluate the methyl ester yield. The $3 / 27$ test and a pHLip test was used to qualitatively determine if there were large quantities of triglycerides (including mono- and di- glycerides) present in the reaction during the final stages of the process (Burton, 2008; Von Wedel, 2006). A ratio of 9 parts methanol to 1 part biodiesel was used for the 3/27 test. In this study, the mass of the biodiesel was measured when no visible settling of oily material or turbidity was observed.

The yield of biodiesel was determined using the following Equation 2 (Azcan and Danisman, 2007; Schinas et al., 2009):

$$
\text { Yield }=\frac{\text { weight of biodiesel }}{\text { weight of feedstock }} \times 100 \%
$$


In the current work, the use of PTA-D led to a maximum yield of $93 \%$ with UCO feedstock and $100 \%$ with palmitic acid. The UCO feedstock contains FFA and glycerides when compared to palmitic acid which is a pure model FFA compound. Therefore, MG, DG and TG were also transesterified in addition to esterification of FFA. Thus, the catalytic activity could vary leading to slight differences in yield observed.

The results show that higher acid loadings lead to higher yield. KIT-5 and frameworkfree PTA were used as controls. KIT-5 framework did not convert FFA's due to its low acidity and ion exchange capability, which limits its effectiveness as a catalyst by itself.

When PTA was loaded, the esterification was effective with yields greater than $90 \%$. When used with low FFA containing oils such as corn oil, the catalyst was inactive. This also proved that the catalyst was effective only with high FFA containing oils owing to its acidic properties.

The percent conversion of FFA to its corresponding fatty acid methyl esters (FAMEs) was calculated by Equation 3 (SathyaSelvabala et al., 2011):

$$
\text { Conversion }=\frac{\text { acid value }_{\text {initial }}-\text { acid value }}{\text { final }} \underset{\text { acid value }}{\text { initial }} \times 100 \%
$$

The maximum conversion of $100 \%$ and $83 \%$ for palmitic acid and UCO was obtained with PTA-D. The acid value of used cooking oil reduced to $2 \mathrm{mg} \mathrm{KOHg}^{-1}$ after acid esterification from an initial value of $11.88 \mathrm{mg}$ of $\mathrm{KOHg}^{-1}$. The percent conversion obtained from all experiments conducted in this study is provided in Figure 5.

In comparison to other studies in the literature, PTA loaded materials were found to be generally more efficient than any other mesoporous catalysts for esterification (Fauzi and Amin, 2013; Olutoye and Hameed, 2011; Zhu et al., 2013). Use of Cs-doped hetero poly tungstates resulted in $100 \%$ palmitic acid conversion after $6 \mathrm{~h}$ of reaction, with $90 \%$ selectivity to the methyl ester and catalysts were recoverable with no leaching of soluble hetero poly tungstates (Narasimharao et al., 2007). Use of $25 \mathrm{wt} \% \mathrm{PTA} / \mathrm{Nb}_{2} \mathrm{O}_{5}$ at conditions of $200{ }^{\circ} \mathrm{C}, 18: 1$ alcohol to oil molar ratio, and $3 \mathrm{wt} \%$ catalyst loading for the transesterification of used cooking oil with high free fatty acids with methanol, resulted in a maximum ester yield of $92 \mathrm{wt} \%$. The catalyst was recycled and reused with negligible loss in activity (Srilatha et al., 2010). 12tungstophosphoric acid (10-40\%) on zeolite $\mathrm{H} \beta$ were synthesized, and $30 \%$ loaded catalyst was used in the esterification of oleic acid with methanol and a $84 \%$ conversion was observed (Patel and Narkhede, 2012). MCM-48-supported hetero poly tungstates catalysts were used in the esterification of long-chain fatty acids and alcohols in supercritical $\mathrm{CO}_{2}$ medium at a temperature of $100{ }^{\circ} \mathrm{C}$ and a short reaction period of $6 \mathrm{~h}$ (Sakthivel et al., 2008). Heterogenized 
heteropolyacids, $\mathrm{H}_{3} \mathrm{PW}_{12} \mathrm{O}_{40} / \mathrm{SiO}_{2}$ and $\mathrm{Cs}_{2} \mathrm{HPW}_{12} \mathrm{O}_{40}$ were shown to catalyze rapeseed oil transesterification with ethanol at $80^{\circ} \mathrm{C}$ and conversions observed was $27 \%, 14 \%$ and $5 \%$ for $\mathrm{H}_{3} \mathrm{PW}_{12} \mathrm{O}_{40}, \mathrm{H}_{3} \mathrm{PW}_{12} \mathrm{O}_{40} / \mathrm{SiO}_{2}$ and $\mathrm{Cs}_{2} \mathrm{HPW}_{12} \mathrm{O}_{40}$ respectively (Hamad et al., 2008). In the esterification reaction of $n$-butanol with acetic acid, both chemically and physically immobilized HPW showed similar catalytic activity around $80 \%$. During repeated uses, rapidly decreasing catalytic activity was observed in the case phosphotungstic acid impregnated palygorskite (Zhang et al., 2010). Duan et al. synthesized $\mathrm{H}_{3} \mathrm{PW}_{12} \mathrm{O}_{40}$ supported on magnetic iron oxide particles, tested them in palmitic acid esterification with methanol and observed a conversion of $90 \%$ at $65^{\circ} \mathrm{C}$ (Duan et al., 2013). CDAB (cetyldimethyl(ethyl)ammonium bromide) and SBA-15 were studied for esterification of oils. SBA-15-SO $\mathrm{S}_{3} \mathrm{H}-\mathrm{P} 123$ was found to be acidic in nature with surface acidity. The biodiesel yield was $85 \%$ after $20 \mathrm{~h}$ of reaction. $\mathrm{CDAB}-\mathrm{SO}_{3} \mathrm{H}$ showed a $55 \%$ yield under similar experimental conditions (Lin and Radu, 2006). Silica supported phosphoric acid was investigated for biodiesel production from triacetin and the conversion was extremely low at less than 10\% (López et al., 2005). MCM-41 was also tested for the transesterification of soybean oil and a yield of $85 \%$ was obtained at $60{ }^{\circ} \mathrm{C}$ using $10 \mathrm{wt} \%$ of catalyst for $24 \mathrm{~h}$ (Georgogianni et al., 2009).

The above discussed solid acid catalysts when investigated for biodiesel synthesis suffered from limitations such as limited catalyst life, difficulty in recyclability, and/or low yields in comparison with the current synthesized catalyst.

\subsection{GC-MS/HPLC Analysis}

The GC-MS (gas chromatography-mass spectrometry) was used to analyze the free fatty acids and methyl esters found in the feedstock and products. Spectra indicated that FFAs such as hexanoic acid, decanoic acid, octanoic acid and eicosanoic acid in the feedstock were converted to their corresponding methyl esters. Figures $6 \mathrm{a}$ and $6 \mathrm{~b}$ indicate the complete conversion of palmitic acid and UCO to biodiesel respectively. Figure 6c shows the spectra of low FFA corn oil at the start and end of the esterification reaction. The two spectra are very similar indicating the absence of any catalytic activity when feedstock contains lower amounts of FFA.

Figure 7 shows a direct comparison in the HPLC chromatograms between the feedstock and the methyl ester formed. The consumption of triglycerides and diglycerides to form fatty acid methyl esters is clearly observed.

\subsection{Recycling studies}

Recycling of the active catalyst is of economic importance for batch processes utilizing catalysts for the production of biodiesel. The main advantage in heterogeneous catalysis is the efficient catalyst recovery. This part of the study shows that this catalyst has been active in the heterogeneous phase, is efficient and can be recovered via a simple recycle protocol. Further, other studies have shown that heteropoly acids anchored to mesoporous supports can be recycled and have been proven to have good recyclability (Ren et al., 2010; Sofia et al., 2009). The 
recyclability of $\mathrm{WO}_{3} / \mathrm{ZrO}_{2}$ was studied under the reaction conditions of $200{ }^{\circ} \mathrm{C}, 3 \mathrm{wt} \%$ catalyst, 5 $\mathrm{h}$ and 15:1 methanol to oil molar ratio. The catalyst was recycled without appreciable loss in activity (Sunita et al., 2008). In another study, a small amount of PTA was found to leach from SBA-15 into liquid phase during the esterification of palmitic, stearic and oleic acid with methanol (Tropecêlo et al., 2010). A similar catalyst, SiPW-8, was used in the production of 1,1diacetates from aldehydes and was successfully recycled for more than 4 cycles with minimal loss in catalytic activity (Wang et al., 2006). Although this is an entirely different application, it helps to signify that these materials are recoverable and recyclable. The result of the four cycle experiment has been summarized in Figure 8. At the end of the fourth cycle, the conversion had decreased to $51 \%$ from $83 \%$, falling about $10 \%$ with each recycle. These are conservative results which will only improve in practice due to the fact that the starting mass of catalyst is very small in these bench scale experiments and thus the recovery might be affected to a larger extent than when starting with a larger mass of the catalyst. Some losses are expected due to leaching of PTA and slow decrease in active surface area of catalysts due to oil adsorption of some sites. While, the conversion and yield was seen to be lower with each recycle, the recovered catalyst did not show any significant deactivation in comparison with similar catalysts which showed around 27\% deactivation in other studies (Sharma et al., 2011) and therefore confirms that these heteropoly acids loaded on high surface area catalysts can be easily recycled for up to 4 cycles. However, recycling efficiency can be improved by using more sophisticated industrial techniques rather than simple laboratory techniques used here. This will also lead to higher conversion after recycling than those reported here. In summary, the most efficient catalyst was PTA-D and important results from the use of that catalyst are summarized in Table 2.

4. Conclusions

Requirements for a good solid acid catalyst for biodiesel synthesis by esterification are (i) high acid concentration to maintain an acceptable reaction rate, (ii) high surface area, and (iii) high conversion and yield. In this study, a mesoporous material based solid acid catalyst, namely PTA loaded KIT-5, was found to satisfy all the above requirements. The highest experimental conversion was $83 \%$ for UCO and $100 \%$ for palmitic acid at the optimum condition of $4 \mathrm{~h}$ reaction time, 2:1 and 6:1 volume ratio of methanol to oil respectively for UCO and palmitic acid, $70{ }^{\circ} \mathrm{C}$ reaction temperature, and $26.5 \mathrm{wt} \%$ catalyst loading. Catalyst recycling studies indicated that a small amount of acid leached out from the framework leading to a decrease in conversion with each recycle. In conclusion, PTA loaded KIT-5 was synthesized, characterized, and was shown to convert high free fatty acid containing feedstock into biodiesel. The kinetic model for the esterification of UCO to biodiesel was established and will be discussed in part II of this article. 
We thank York Smith for technical support with XRD and BET (surface area) measurements and Dr. V. V. Balasubramaniam for initial discussions regarding this work. This research was funded by the Department of Energy under grant number DE-EE0003158.

\section{References}

Ahmed, S., Hassan, M.H., Kalam, M.A., Ashrafur Rahman, S.M., Abedin, M.J., Shahir, A., 2014. An experimental investigation of biodiesel production, characterization, engine performance, emission and noise of Brassica juncea methyl ester and its blends. Journal of Cleaner Production 79, 74-81.

Alsalme, A., Kozhevnikova, E.F., Kozhevnikov, I.V., 2008. Heteropoly acids as catalysts for liquid-phase esterification and transesterification. Applied Catalysis A: General 349, 170-176.

AOCS, Method Ca 5a-40: Free Fatty Acids, Firestone, D. (Eds.), Official Methods and Recommended Practices of the American Oil Chemists' Society, fifth edition, American Oil Chemists' Society, Champaign, IL, USA, (1998).

Azcan, N., Danisman, A., 2007. Alkali catalyzed transesterification of cottonseed oil by microwave irradiation. Fuel 86, 2639-2644.

Bardin, B.B., Davis, R.J., 2000. Effect of water on silica-supported phosphotungstic acid catalysts for 1-butene double bond shift and alkane skeletal isomerization. Applied Catalysis A: General 200, 219-231.

Burton, R., 2008. An overview of ASTM D6751: biodiesel standards and testing methods. Alternative Fuels Consortium.

Cho, H.J., Kim, S.H., Hong, S.W., Yeo, Y.-K., A single step non-catalytic esterification of palm fatty acid distillate (PFAD) for biodiesel production. Fuel 93, 373-380.

Di Serio, M., Cozzolino, M., Giordano, M., Tesser, R., Patrono, P., Santacesaria, E., 2007. From Homogeneous to Heterogeneous Catalysts in Biodiesel Production. Industrial \& Engineering Chemistry Research 46, 6379-6384.

Dovì, V.G., Friedler, F., Huisingh, D., Klemeš, J.J., 2009. Cleaner energy for sustainable future. Journal of Cleaner Production 17, 889-895.

Duan, X., Liu, Y., Zhao, Q., Wang, X., Li, S., 2013. Water-tolerant heteropolyacid on magnetic nanoparticles as efficient catalysts for esterification of free fatty acid. RSC Advances 3, 1374813755.

Fauzi, A.H.M., Amin, N.A.S., 2013. Catalysis in Biodiesel Synthesis: Challenges and Future Perspectives, Advances in Biofuels. Springer, pp. 127-152.

Georgogianni, K.G., Katsoulidis, A.P., Pomonis, P.J., Kontominas, M.G., 2009. Transesterification of soybean frying oil to biodiesel using heterogeneous catalysts. Fuel Processing Technology 90, 671-676.

Gerpen, J.V., 2005. Biodiesel processing and production. Fuel Processing Technology 86, 10971107. 
Hamad, B., Lopes de Souza, R.O., Sapaly, G., Carneiro Rocha, M.G., Pries de Oliveira, P.G., Gonzalez, W.A., Andrade Sales, E., Essayem, N., 2008. Transesterification of rapeseed oil with ethanol over heterogeneous heteropolyacids. Catalysis Communications 10, 92-97.

Hough, T.P., 2007. Recent Developments in Solar Energy. Nova Science Publishers, 150-154.

Jagadeeswaraiah, K., Balaraju, M., Prasad, P.S.S., Lingaiah, N., 2010. Selective esterification of glycerol to bioadditives over heteropoly tungstate supported on Cs-containing zirconia catalysts. Applied Catalysis A: General 386, 166-170.

Kleitz, F., Liu, D., Anilkumar, G.M., Park, I.-S., Solovyov, L.A., Shmakov, A.N., Ryoo, R., 2003. Large Cage Face-Centered-Cubic Fm3m Mesoporous Silica: Synthesis and Structure. The Journal of Physical Chemistry B 107, 14296-14300.

Knothe, G., Steidley, K.R., 2009. A comparison of used cooking oils: A very heterogeneous feedstock for biodiesel. Bioresource Technology 100, 5796-5801.

Kondamudi, N., Mohapatra, S.K., Misra, M., 2011. Quintinite as a bifunctional heterogeneous catalyst for biodiesel synthesis. Applied Catalysis A: General 393, 36-43.

Kozhevnikov, I.V., 1998. Catalysis by Heteropoly Acids and Multicomponent Polyoxometalates in Liquid-Phase Reactions. Chemical Reviews 98, 171-198.

Kumar, R., Ravi Kumar, G., Chandrashekar, N., 2011. Microwave assisted alkali-catalyzed transesterification of Pongamia pinnata seed oil for biodiesel production. Bioresource Technology 102, 6617-6620.

Leung, D.Y.C., Guo, Y., 2006. Transesterification of neat and used frying oil: Optimization for biodiesel production. Fuel Processing Technology 87, 883-890.

Leung, D.Y.C., Wu, X., Leung, M.K.H., 2010. A review on biodiesel production using catalyzed transesterification. Applied Energy 87, 1083-1095.

Lin, V.S.-Y., Radu, D.R., 2006. Use of functionalized mesoporous silicates to esterify fatty acids and transesterify oils. US Patent 7122688 B2, Oct 17, 2006.

López-Suárez, F.E., Bueno-López, A., Illán-Gómez, M.J., 2008. Cu/ $\mathrm{Al}_{2} \mathrm{O}_{3}$ catalysts for soot oxidation: Copper loading effect. Applied Catalysis B: Environmental 84, 651-658.

López, D.E., Goodwin Jr, J.G., Bruce, D.A., Lotero, E., 2005. Transesterification of triacetin with methanol on solid acid and base catalysts. Applied Catalysis A: General 295, 97-105.

Lynch, J., 2003. Physico-chemical analysis of industrial catalysts: a practical guide to characterization. Editions Technip, University of Michigan, 53-62.

Ma, F., Hanna, M.A., 1999. Biodiesel production: a review. Bioresource Technology 70, 1-15.

Marchetti, J.M., Miguel, V.U., Errazu, A.F., 2007. Possible methods for biodiesel production. Renewable and Sustainable Energy Reviews 11, 1300-1311.

Meng, X., Chen, G., Wang, Y., 2008. Biodiesel production from waste cooking oil via alkali catalyst and its engine test. Fuel Processing Technology 89, 851-857.

Mushrush, G.W., Beal, E.J., Mose, D.G., McClelland, D.B., 1991. The pyrolysis reactions and jet fuel potential of a Green River shale oil. Journal of Analytical and Applied Pyrolysis 22, 7382. 
Nair, P., Singh, B., Upadhyay, S.N., Sharma, Y.C., 2012. Synthesis of biodiesel from low FFA waste frying oil using calcium oxide derived from Mereterix mereterix as a heterogeneous catalyst. Journal of Cleaner Production 29-30, 82-90.

Narasimharao, K., Brown, D.R., Lee, A.F., Newman, A.D., Siril, P.F., Tavener, S.J., Wilson, K., 2007. Structure-activity relations in Cs-doped heteropolyacid catalysts for biodiesel production. Journal of Catalysis 248, 226-234.

Nie, K., Xie, F., Wang, F., Tan, T., 2006. Lipase catalyzed methanolysis to produce biodiesel: Optimization of the biodiesel production. Journal of Molecular Catalysis B: Enzymatic 43, 142147.

Olutoye, M., Hameed, B., 2011. Synthesis of fatty acid methyl ester from crude jatropha (Jatropha curcas Linnaeus) oil using aluminium oxide modified $\mathrm{Mg}-\mathrm{Zn}$ heterogeneous catalyst. Bioresource technology 102, 6392-6398.

Patel, A., Narkhede, N., 2012. 12-Tungstophosphoric Acid Anchored to Zeolite Hß: Synthesis, Characterization, and Biodiesel Production by Esterification of Oleic Acid with Methanol. Energy \& Fuels 26, 6025-6032.

Peña-Alonso, R., Rubio, J., Rubio, F., Oteo, J.L., 2002. A FT-IR Study of the Synthesis of Boron Ormosils by Means of the Sol-Gel Process. Journal of Sol-Gel Science and Technology 25, 255263.

Ramos, M.J., Fernández, C.M., Casas, A., Rodríguez, L., Pérez, Á., 2009. Influence of fatty acid composition of raw materials on biodiesel properties. Bioresource Technology 100, 261-268.

Ren, Y., Yue, B., Gu, M., He, H., 2010. Progress of the application of mesoporous silicasupported heteropolyacids in heterogeneous catalysis and preparation of nanostructured metal oxides. Materials 3, 764-785.

Sakai, T., Kawashima, A., Koshikawa, T., 2009. Economic assessment of batch biodiesel production processes using homogeneous and heterogeneous alkali catalysts. Bioresource Technology 100, 3268-3276.

Sakthivel, A., Komura, K., Sugi, Y., 2008. MCM-48 Supported Tungstophosphoric Acid: An Efficient Catalyst for the Esterification of Long-Chain Fatty Acids and Alcohols in Supercritical Carbon Dioxide. Industrial \& Engineering Chemistry Research 47, 2538-2544.

SathyaSelvabala, V., Selvaraj, D.K., Kalimuthu, J., Periyaraman, P.M., Subramanian, S., 2011. Two-step biodiesel production from Calophyllum inophyllum oil: Optimization of modified $\beta$ zeolite catalyzed pre-treatment. Bioresource technology 102, 1066-1072.

Schinas, P., Karavalakis, G., Davaris, C., Anastopoulos, G., Karonis, D., Zannikos, F., Stournas, S., Lois, E., 2009. Pumpkin (Cucurbita pepo L.) seed oil as an alternative feedstock for the production of biodiesel in Greece. Biomass and Bioenergy 33, 44-49.

Sharma, Y.C., Singh, B., 2009. Development of biodiesel: Current scenario. Renewable and Sustainable Energy Reviews 13, 1646-1651.

Sharma, Y.C., Singh, B., Korstad, J., 2011. Advancements in solid acid catalysts for ecofriendly and economically viable synthesis of biodiesel. Biofuels, Bioproducts and Biorefining 5, 69-92. 
Singh, S., Patel, A., 2014. 12-Tungstophosphoric acid supported on mesoporous molecular material: synthesis, characterization and performance in biodiesel production. Journal of Cleaner Production 72, 46-56.

Singh, S., Patel, A., 2014. Selective Green Esterification and Oxidation of Glycerol over 12Tungstophosphoric Acid Anchored to MCM-48. Industrial \& Engineering Chemistry Research $53,14592-14600$.

Sofia, L.T.A., Krishnan, A., Sankar, M., Kala Raj, N.K., Manikandan, P., Rajamohanan, P.R., Ajithkumar, T.G., 2009. Immobilization of Phosphotungstic Acid (PTA) on Imidazole Functionalized Silica: Evidence for the Nature of PTA Binding by Solid State NMR and Reaction Studies. The Journal of Physical Chemistry C 113, 21114-21122.

Srilatha, K., Issariyakul, T., Lingaiah, N., Sai Prasad, P.S., Kozinski, J., Dalai, A.K., 2010. Efficient Esterification and Transesterification of Used Cooking Oil Using 12Tungstophosphoric Acid (TPA)/ $\mathrm{Nb}_{2} \mathrm{O}_{5}$ Catalyst. Energy \& Fuels 24, 4748-4755.

Staiti, P., Freni, S., Hocevar, S., 1999. Synthesis and characterization of proton-conducting materials containing dodecatungstophosphoric and dodecatungstosilic acid supported on silica. Journal of Power Sources 79, 250-255.

Sunita, G., Devassy, B.M., Vinu, A., Sawant, D.P., Balasubramanian, V.V., Halligudi, S.B., 2008. Synthesis of biodiesel over zirconia-supported isopoly and heteropoly tungstate catalysts. Catalysis Communications 9, 696-702.

Tan, R., Liu, C., Feng, N., Xiao, J., Zheng, W., Zheng, A., Yin, D., 2012. Phosphotungstic acid loaded on hydrophilic ionic liquid modified SBA-15 for selective oxidation of alcohols with aqueous $\mathrm{H}_{2} \mathrm{O}_{2}$. Microporous and Mesoporous Materials 158, 77-87.

Tang, Y., Xu, J., Zhang, J., Lu, Y., 2013. Biodiesel production from vegetable oil by using modified $\mathrm{CaO}$ as solid basic catalysts. Journal of Cleaner Production 42, 198-203.

Tropecêlo, A.I., Casimiro, M.H., Fonseca, I.M., Ramos, A.M., Vital, J., Castanheiro, J.E., 2010. Esterification of free fatty acids to biodiesel over heteropolyacids immobilized on mesoporous silica. Applied Catalysis A: General 390, 183-189.

Von Wedel, R., 2006. Quantitative Studies of the pHLip Test Kit for B100, Biodiesel Technical Workshop, Tucson.

Wan Ghazali, W.N.M., Mamat, R., Masjuki, H.H., Najafi, G., 2015. Effects of biodiesel from different feedstocks on engine performance and emissions: A review. Renewable and Sustainable Energy Reviews 51, 585-602.

Wang, J., Groen, J.C., Coppens, M.-O., 2008. Unified Formation Mechanism of Disordered Mesoporous Silica, Structured by Means of Nontemplating Organic Additives. The Journal of Physical Chemistry C 112, 19336-19345.

Wang, J., Yan, L., Qian, G., Yang, K., Liu, H., Wang, X., 2006. Heteropolyacid encapsulated into mesoporous silica framework for an efficient preparation of 1,1-diacetates from aldehydes under a solvent-free condition. Tetrahedron Letters 47, 8309-8312.

Zhang, J., Jiang, L., 2008. Acid-catalyzed esterification of Zanthoxylum bungeanum seed oil with high free fatty acids for biodiesel production. Bioresource Technology 99, 8995-8998. 
1 Zhang, L., Jin, Q., Shan, L., Liu, Y., Wang, X., Huang, J., 2010. $\mathrm{H}_{3} \mathrm{PW}_{12} \mathrm{O}_{40}$ immobilized on 2 silylated palygorskite and catalytic activity in esterification reactions. Applied Clay Science 47, 3 229-234.

4 Zhang, Y., Dubé, M.A., McLean, D.D., Kates, M., 2003. Biodiesel production from waste 5 cooking oil: 1. Process design and technological assessment. Bioresource Technology 89, 1-16.

6 Zhu, S., Gao, X., Dong, F., Zhu, Y., Zheng, H., Li, Y., 2013. Design of a highly active silver7 exchanged phosphotungstic acid catalyst for glycerol esterification with acetic acid. Journal of 8 Catalysis 306, 155-163. 


\section{Table captions}

Table 1. Surface area and amount of phosphotungstic acid loaded on to the framework of various catalysts used. KIT-5 refers to framework with no catalyst and catalyst with notations 'PTA' refer to PTA loaded KIT-5. The large specific surface area of the catalyst ensured good contact between the reactant molecules and catalytic active sites.

Table 2. Summary of results obtained from the use of catalyst PTA-D, which was found to be the most effective catalyst in this study.

\section{Figure captions}

Figure 1. FTIR Spectra of KIT-5, PTA and PTA loaded on KIT-5. The symmetrical/symmetrical stretching corresponding to $\mathrm{Si}-\mathrm{O}-\mathrm{Si}$ bonds and $\mathrm{Si}-\mathrm{OH}$ vibrations of surface silanols that are characteristic of mesoporous silica are shown.

Figure 2. SEM image of (a) PTA-A, (b) PTA-B, (c) PTA-C, (d) PTA-D. The morphology and size of the catalyst particles were as expected. Agglomerations of small spherical particles were observed. There was negligible difference in particle surface morphology between the KIT-5 host and PTA exchanged KIT-5.

Figure 3. XRD patterns of PTA, KIT-5 framework and PTA incorporated KIT-5. Increasing amount of PTA in KIT-5 can be seen to lead to an increase in the intensity of the PTA peaks in the loaded catalysts.

Figure 4. $\mathrm{N}_{2}$ adsorption/desorption isotherms of (a) KIT-5 (b) PTA-A (c) PTA-B (d) PTA-C (e) PTA-D. KIT-5 and PTA loaded KIT-5 exhibit Type IV/Type V mesoporous behavior, which is typically observed for the mesoporous samples with a cage-type pore structure.

Figure 5. Percent conversion of used cooking oil and palmitic acid to FAME obtained with various catalysts. While use of palmitic acid is seen to result in $100 \%$ conversion, use of used cooking oil only leads to a maximum of $83 \%$ conversion.

Figure 6 (a) GCMS spectra of UCO prior to and after conversion using PTA-D. All identified components have been labeled. (b) GCMS spectra show methyl ester formation when palmitic acid was used as a feedstock on reaction with PTA-D and PTA; Spectra of pure palmitic acid is provided for comparison. (c) GCMS spectra show that PTA loaded KIT-5 catalyst is unable to convert oils with low FFA.

Figure 7. HPLC system allowed for the detection of mono-, di- and tri-glycerides as well as methyl esters. The difference between the feedstock and the biodiesel is clearly demonstrated in which the feedstock contains more mono-, di- and tri-glycerides and the biodiesel sample contains a small quantity of unreacted tri-glycerides and methyl esters. 
Figure 8: Yield and conversion of used cooking oil to biodiesel from recycling studies for each run. 
Table 1. Surface area and amount of phosphotungstic acid loaded on to the framework of various catalysts used. KIT-5 refers to framework with no catalyst and catalyst with notations 'PTA' refer to PTA loaded KIT-5. The large specific surface area of the catalyst ensured good contact between the reactant molecules and catalytic active sites.

\begin{tabular}{ccc}
\hline Desired Catalyst & $\begin{array}{c}\text { Amount of acid loaded on } \\
\text { framework }\end{array}$ & $\begin{array}{c}\text { Surface area } \\
\left(\mathrm{m}^{2} \mathrm{~g}^{-1}\right)\end{array}$ \\
\hline KIT-5 & - & 978 \\
PTA-A & $4.1 \%$ & 574 \\
PTA-B & $7.8 \%$ & 525 \\
PTA-C & $10.6 \%$ & 447 \\
PTA-D & $26.5 \%$ & 425 \\
\hline
\end{tabular}


Table 2. Summary of results obtained from the use of catalyst PTA-D, which was found to be the most effective catalyst in this study.

\begin{tabular}{llr}
\hline & Catalyst & - PTA-D \\
\hline $\begin{array}{l}\text { Mass of PTA loaded on KIT-5 } \\
\text { framework }\end{array}$ & $26.5 \mathrm{wt} \%$ \\
Surface Area & & \\
Yield & $425 \mathrm{~m}^{2} \mathrm{~g}^{-1}$ & \\
& Palmitic acid & $100 \%$ \\
Conversion & UCO & $93 \%$ \\
& Palmitic acid & $83 \%$ \\
Recyclability & UCO & \\
& Recycled for 4 cycles with $20 \%$ loss in \\
& yield and 22\% loss in conversion at the \\
\hline
\end{tabular}




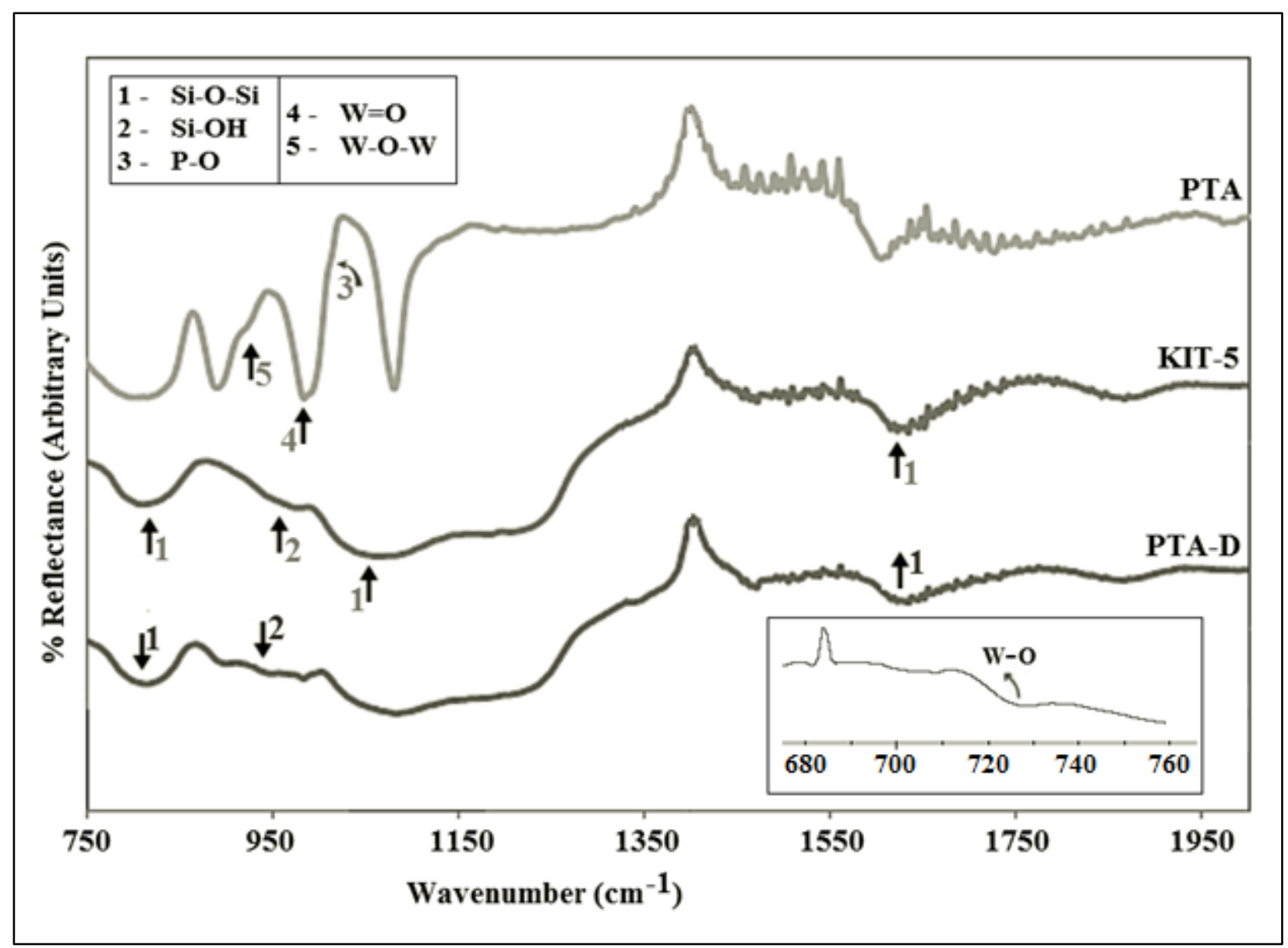

Figure 1. FTIR Spectra of KIT-5, PTA and PTA loaded on KIT-5. The symmetrical/symmetrical stretching corresponding to $\mathrm{Si}-\mathrm{O}-\mathrm{Si}$ bonds and $\mathrm{Si}-\mathrm{OH}$ vibrations of surface silanols that are characteristic of mesoporous silica are shown. 


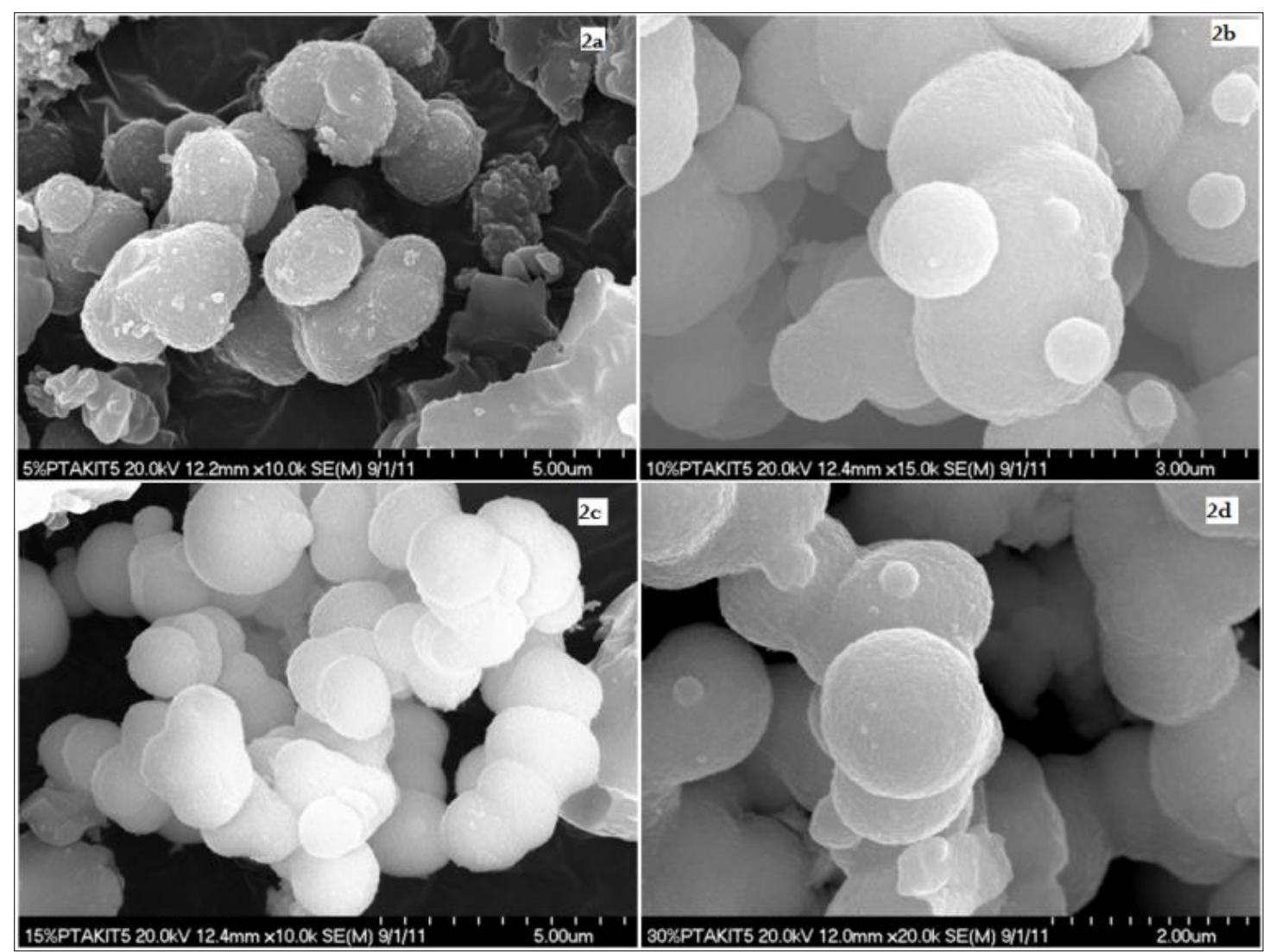

Figure 2. SEM image of (a) PTA-A, (b) PTA-B, (c) PTA-C, (d) PTA-D. The morphology and size of the catalyst particles were as expected. Agglomerations of small spherical particles were observed. There was negligible difference in particle surface morphology between the KIT-5 host and PTA exchanged KIT-5. 


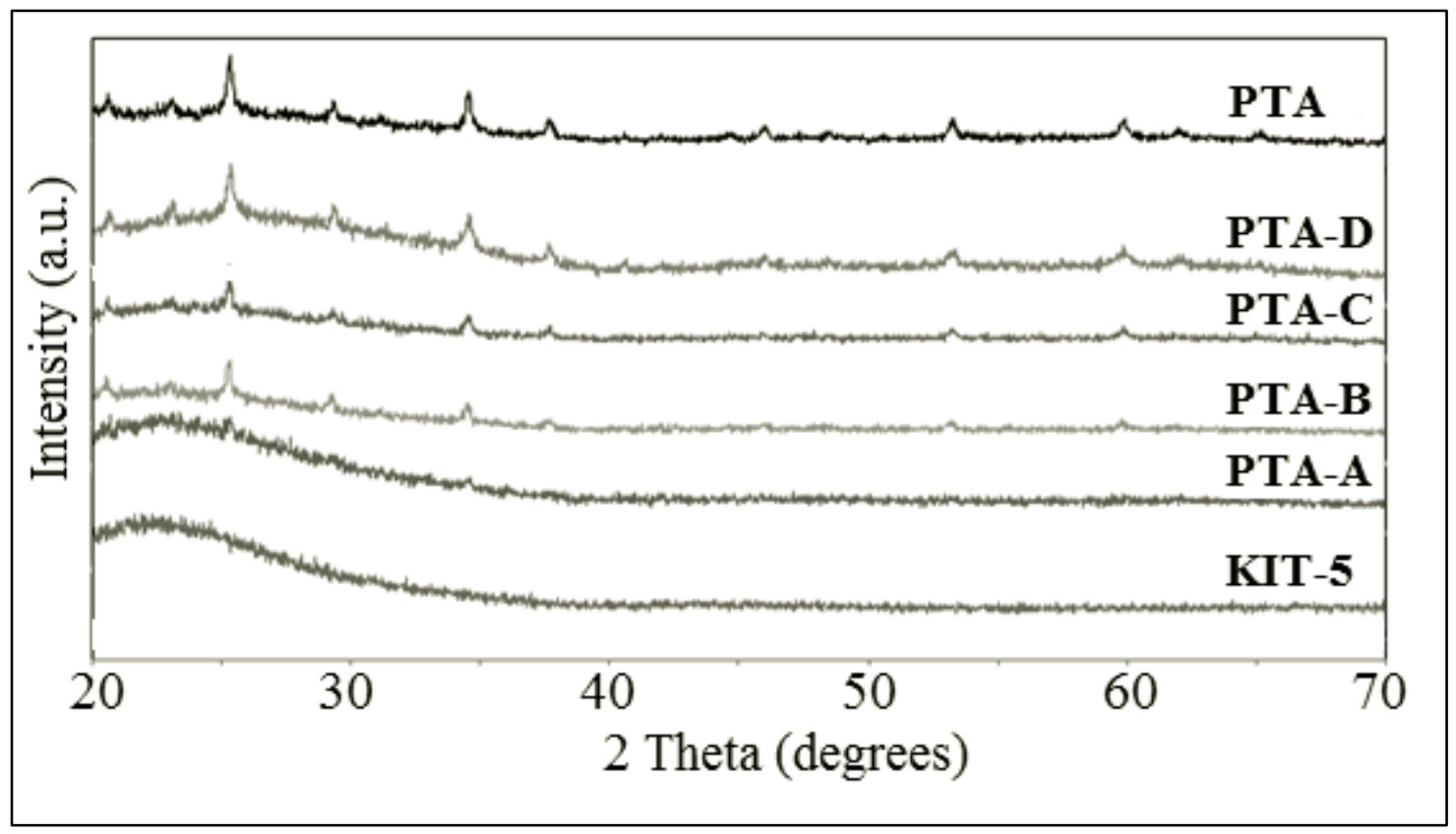

Figure 3. XRD patterns of PTA, KIT-5 framework and PTA incorporated KIT-5. Increasing amount of PTA in KIT-5 can be seen to lead to an increase in the intensity of the PTA peaks in the loaded catalysts. 


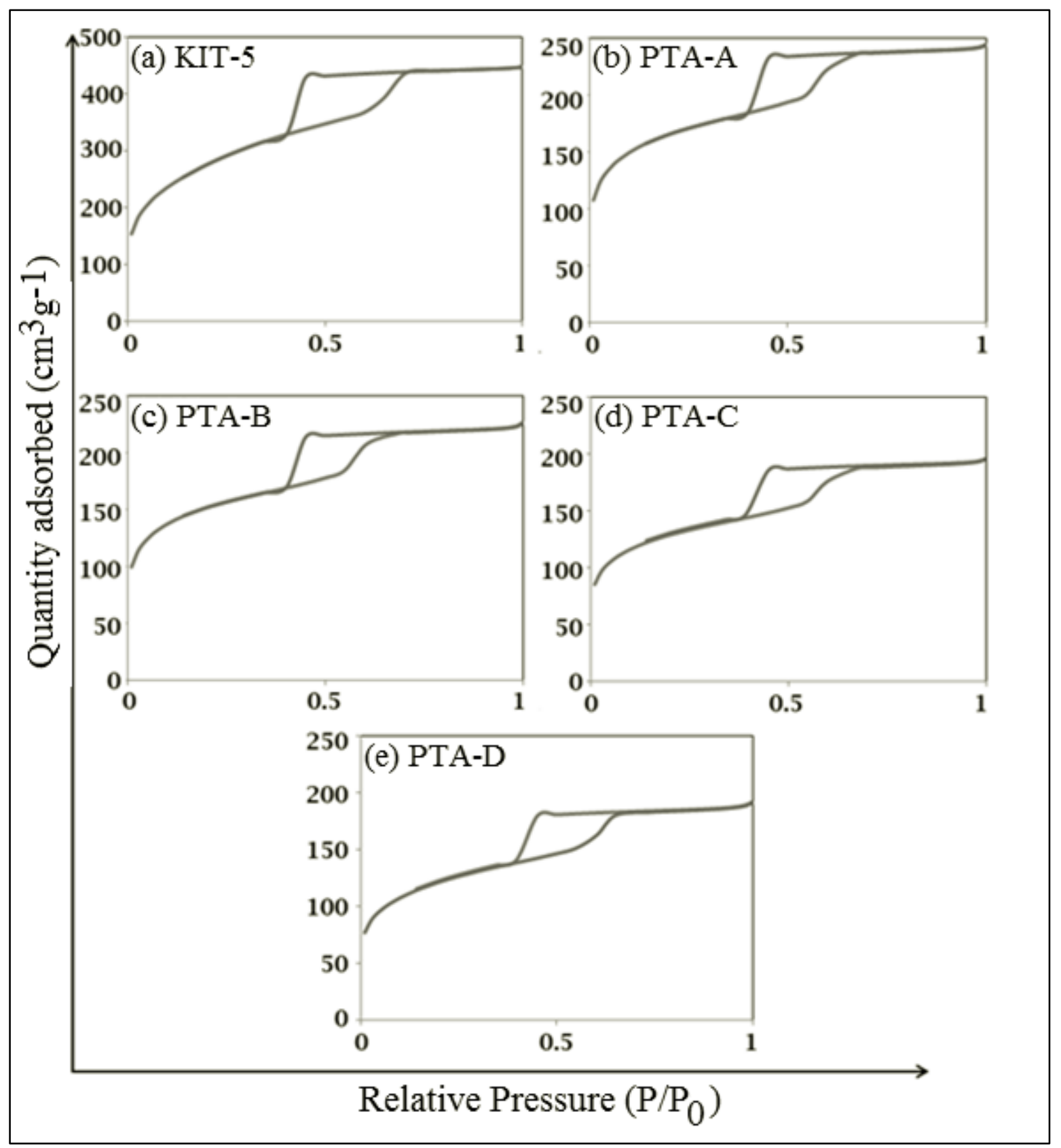

Figure 4. $\mathrm{N}_{2}$ adsorption/desorption isotherms of (a) KIT-5 (b) PTA-A (c) PTA-B (d) PTA-C (e) PTA-D. KIT-5 and PTA loaded KIT-5 exhibit Type IV/Type V mesoporous behavior, which is typically observed for the mesoporous samples with a cage-type pore structure. 


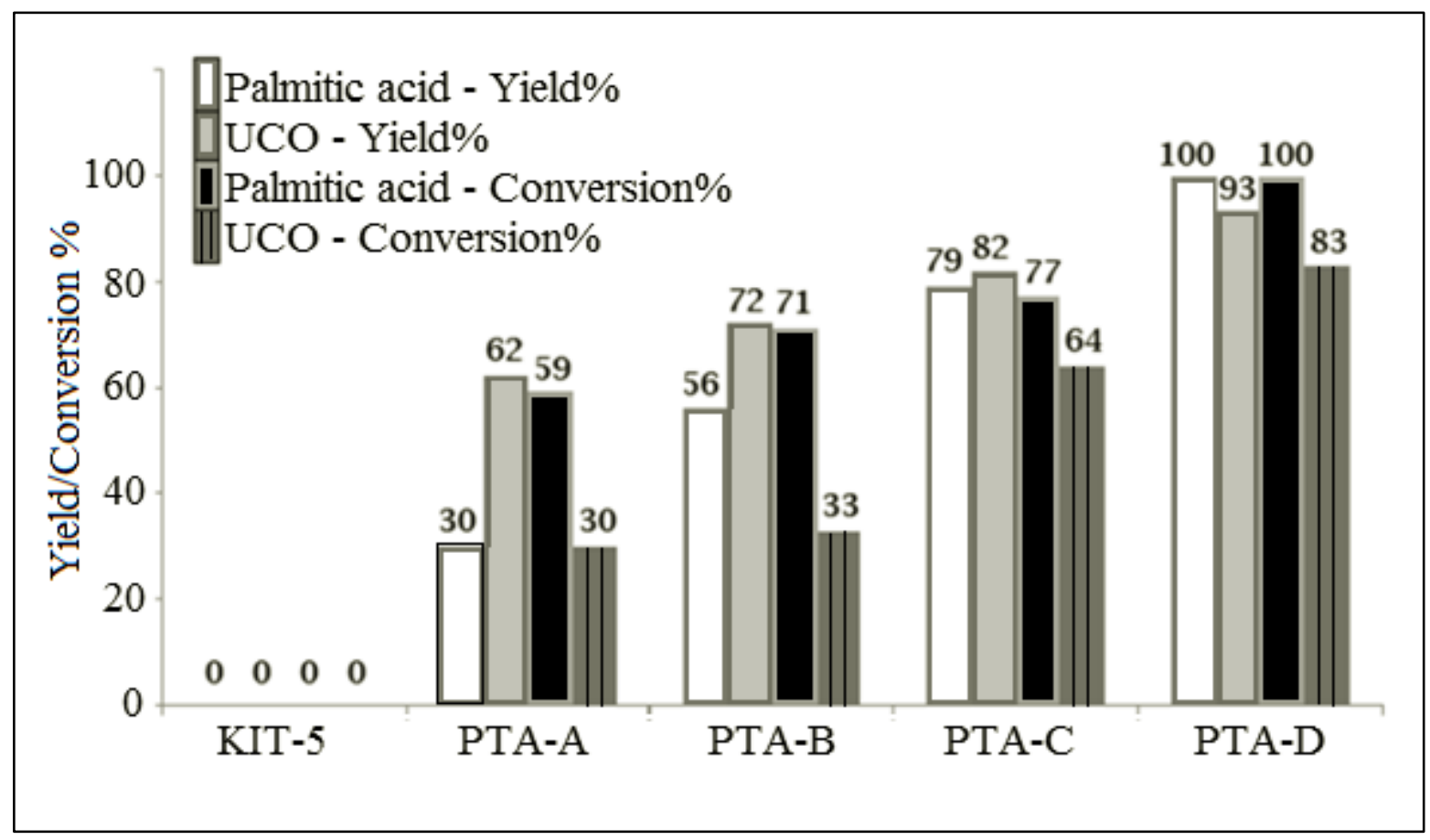

Figure 5. Percent conversion of used cooking oil and palmitic acid to FAME obtained with various catalysts. While use of palmitic acid is seen to result in $100 \%$ conversion, use of used cooking oil only leads to a maximum of $83 \%$ conversion. 

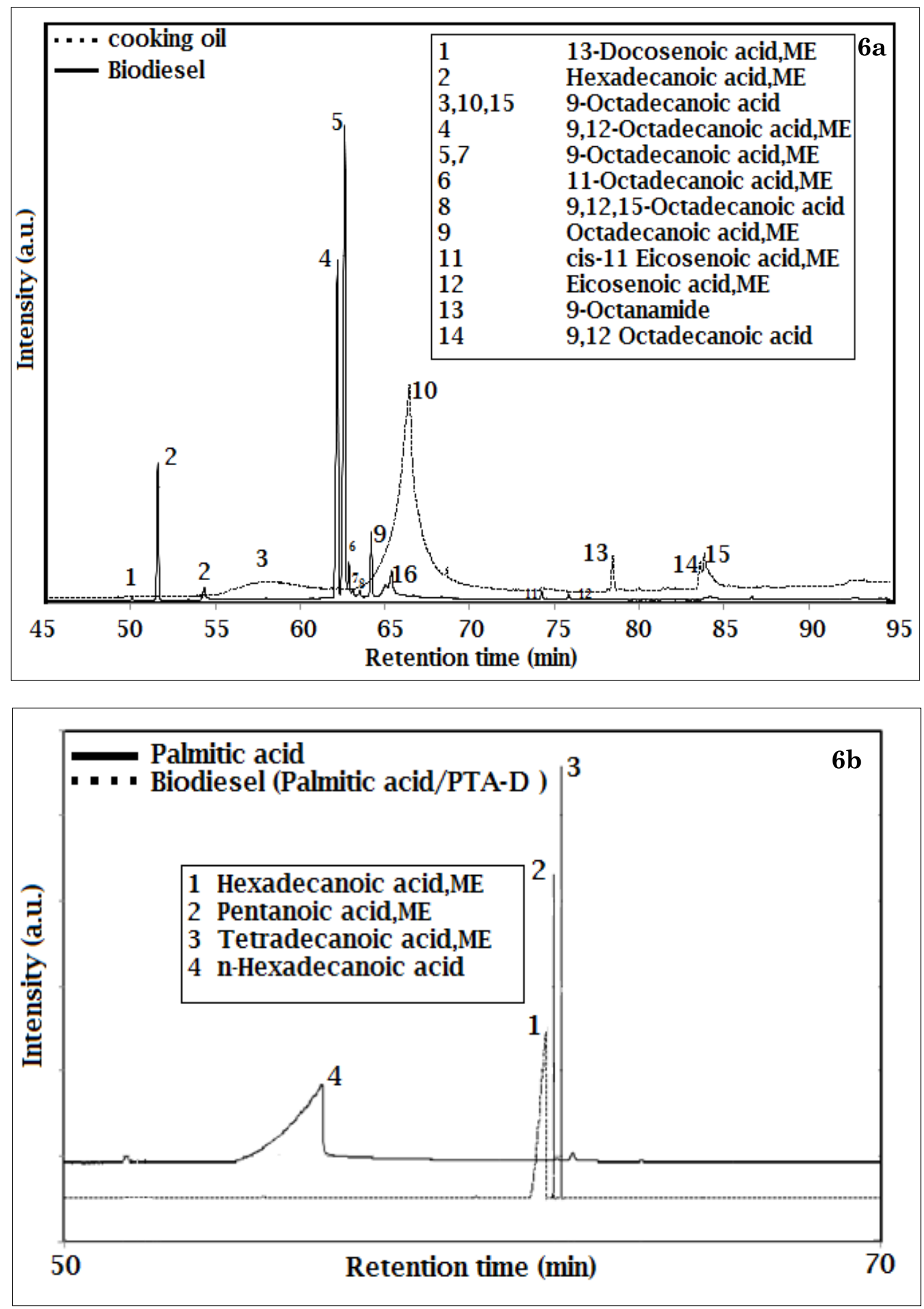


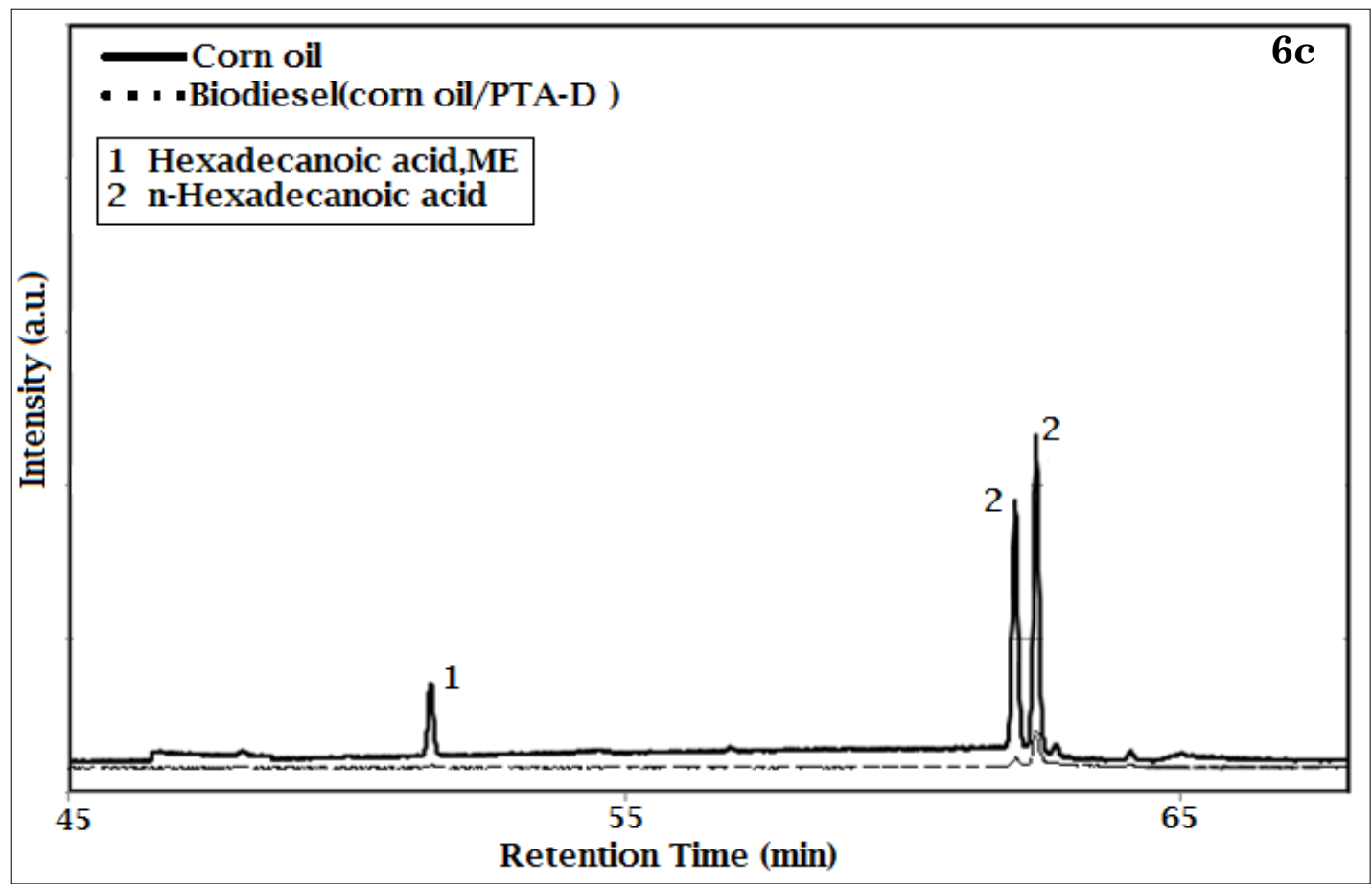

Figure 6 (a) GCMS spectra of UCO prior to and after conversion using PTA-D. All identified components have been labeled. (b) GCMS spectra show methyl ester formation when palmitic acid was used as a feedstock on reaction with PTA-D and PTA; Spectra of pure palmitic acid is provided for comparison. (c) GCMS spectra show that PTA loaded KIT-5 catalyst is unable to convert oils with low FFA. 


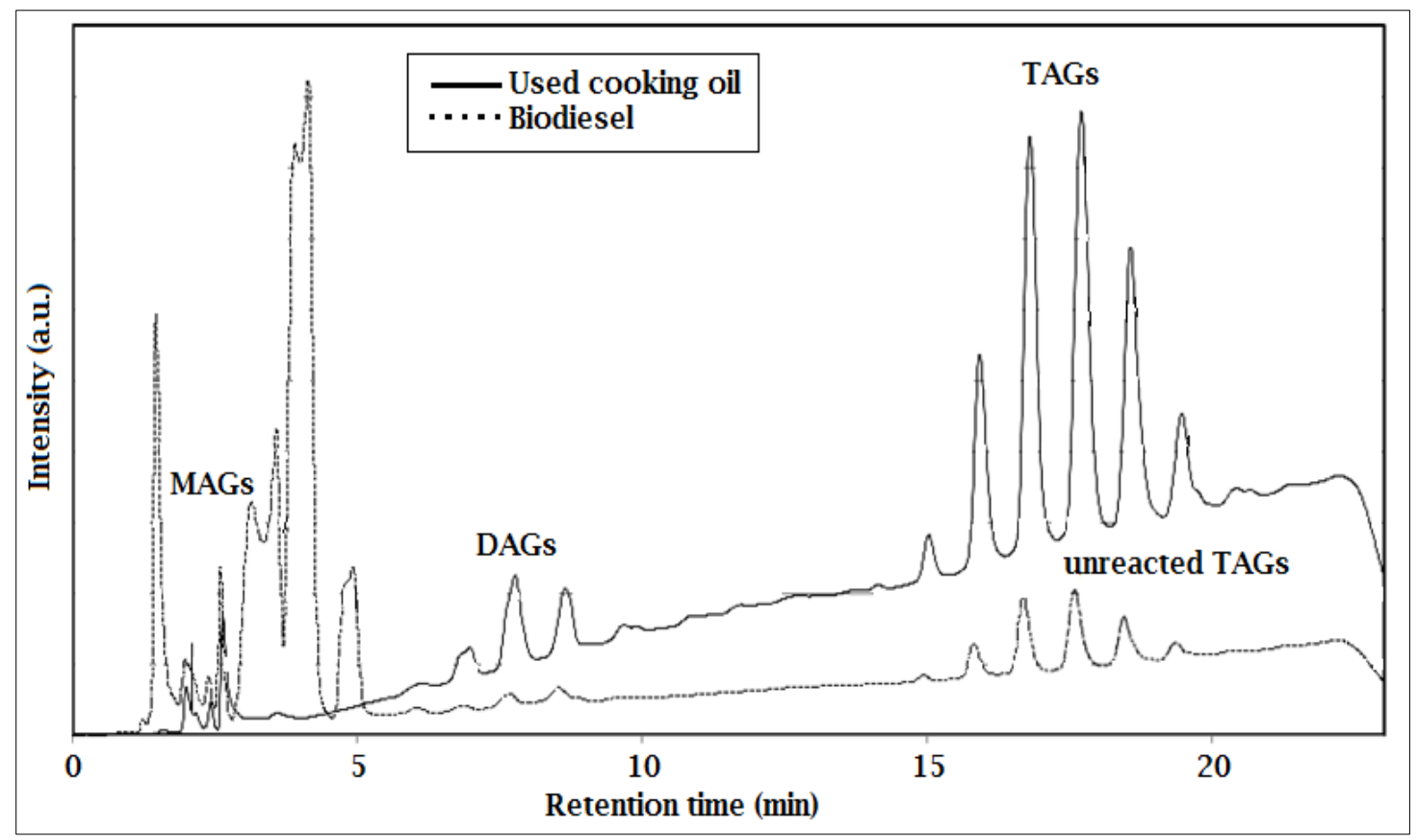

Figure 7. HPLC system allowed for the detection of mono-, di- and tri-glycerides as well as methyl esters. The difference between the feedstock and the biodiesel is clearly demonstrated in which the feedstock contains more mono-, di- and tri-glycerides and the biodiesel sample contains a small quantity of unreacted tri-glycerides and methyl esters. 


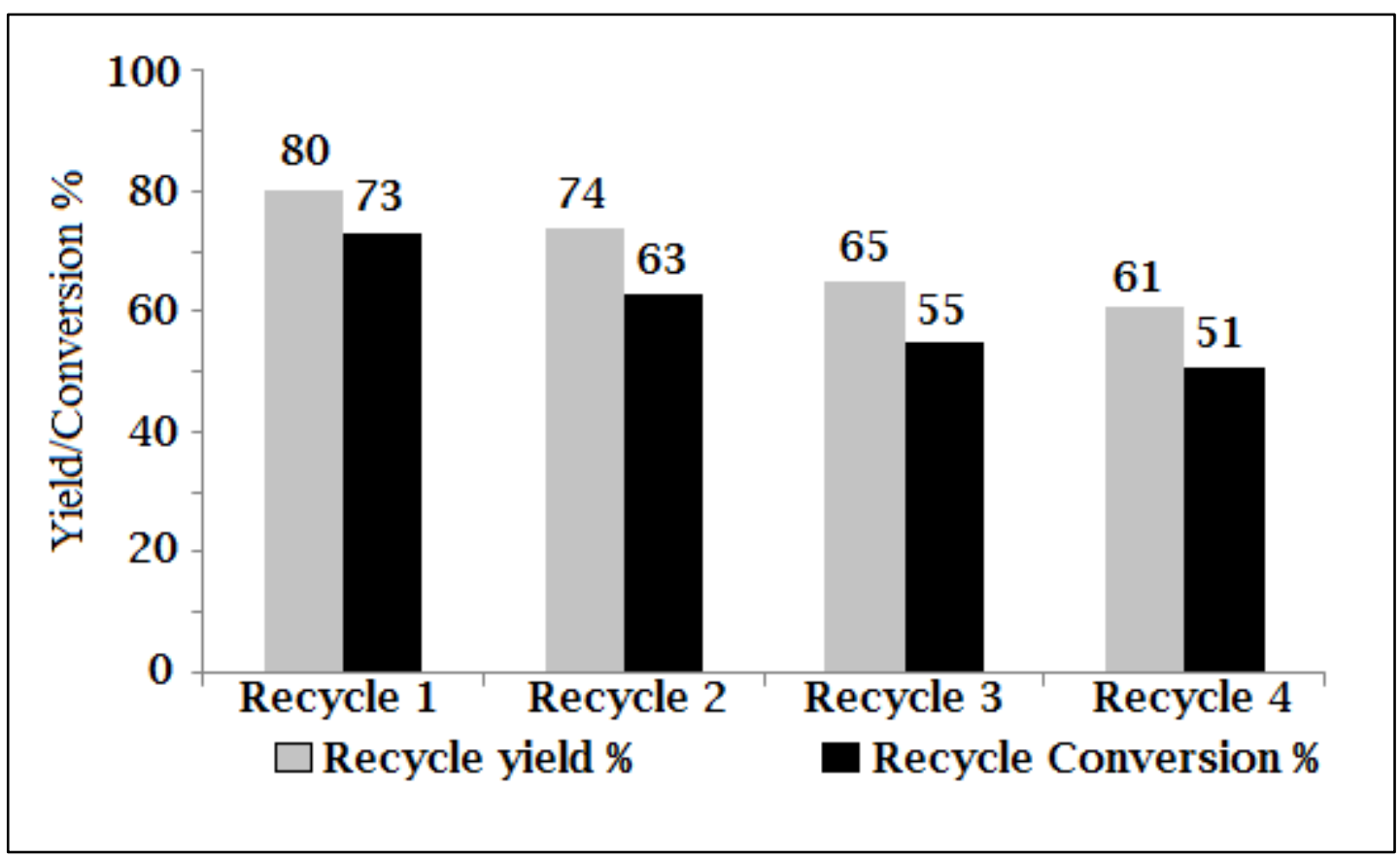

Figure 8: Yield and conversion of used cooking oil to biodiesel from recycling studies for each run. 
- Silica framework synthesized

- Phosphotungstic acid impregnated on the framework

- Material morphology and chemistry characterization
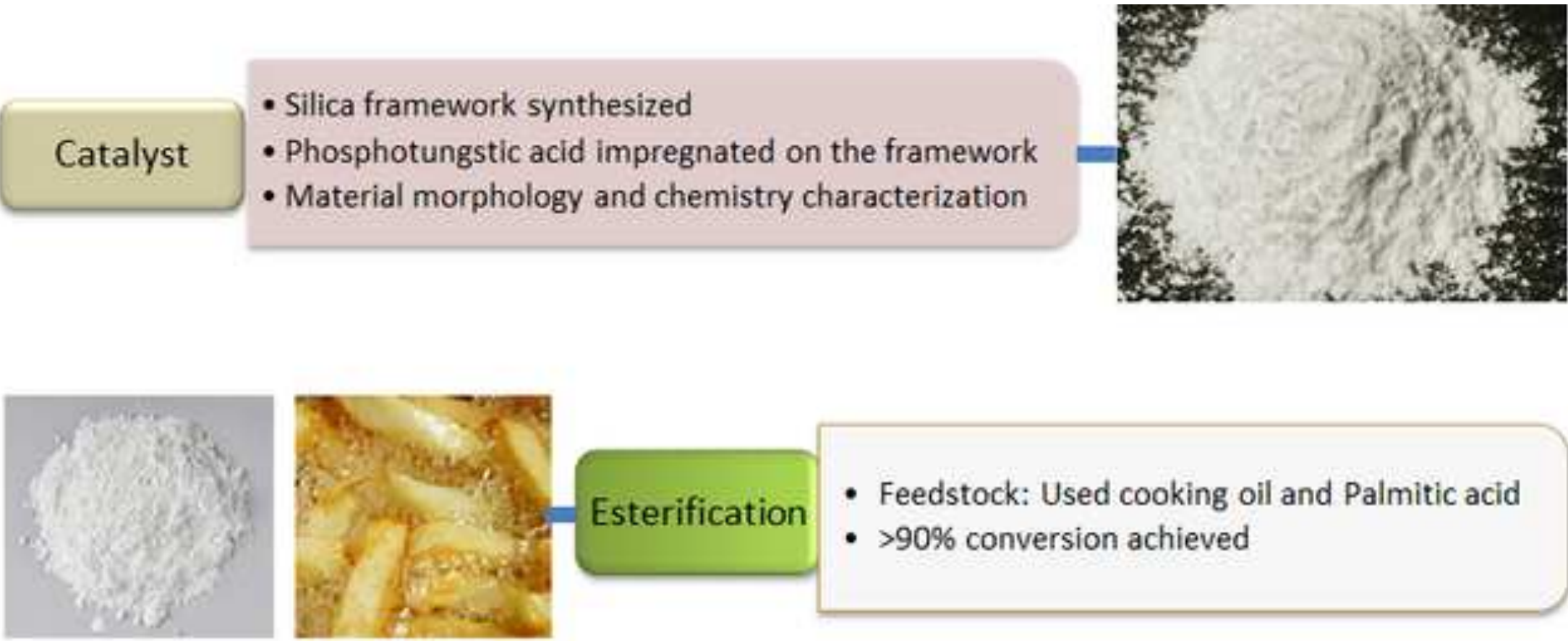

- Feedstock: Used cooking oil and Palmitic acid

- $>90 \%$ conversion achieved

Step 3

\section{Studies}

- Catalyst leaching and recycling studies

- Biodiesel quantification and methyl esters analysis

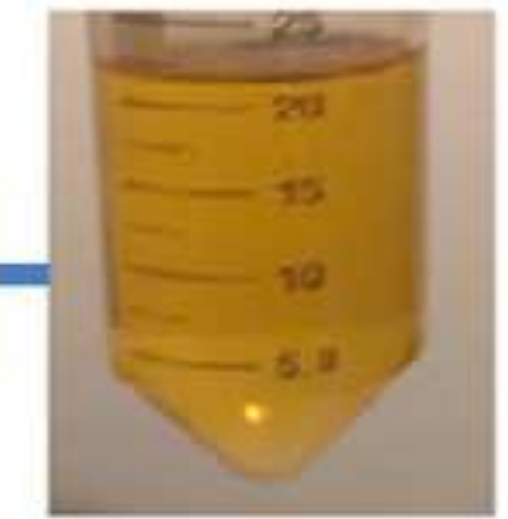

